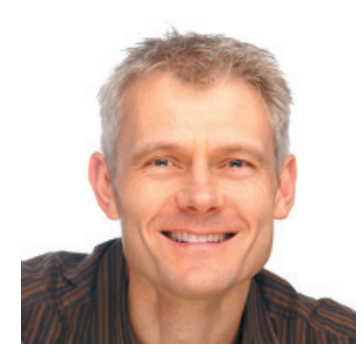

\title{
Talks in the city of light generate more heat
}

\author{
Rather than relying on far-off negative-emissions technologies, Paris needed \\ to deliver a low-carbon road map for today, argues Kevin Anderson.
}

$\mathrm{T}$ The climate agreement delivered earlier this month in Paris is a genuine triumph of international diplomacy. It is a tribute to how France was able to bring a fractious world together. And it is testament to how assiduous and painstaking science can defeat the unremitting programme of misinformation that is perpetuated by powerful vested interests. It is the twenty-first century's equivalent to the victory of heliocentrism over the inquisition. Yet it risks being total fantasy.

Let's be clear, the international community not only acknowledged the seriousness of climate change, it also demonstrated sufficient unanimity to define it quantitatively: to hold "the increase in ... temperature to well below $2{ }^{\circ} \mathrm{C} \ldots$ and to pursue efforts to limit the temperature increase to $1.5^{\circ} \mathrm{C}$ ".

To achieve such goals demands urgent and significant cuts in emissions. But rather than requiring that nations reduce emissions in the short-to-medium term, the Paris agreement instead rests on the assumption that the world will successfully suck the carbon pollution it produces back from the atmosphere in the longer term. A few years ago, these exotic Dr Strangelove options were discussed only as last-ditch contingencies. Now they are Plan A.

Governments, prompted by their advisers, have plumped for BECCS (biomass energy carbon capture and storage) as the most promising 'negativeemissions technology'.

What does BECCS entail? Apportioning huge swathes of the planet's landmass to the growing of bioenergy crops (from big trees to tall grasses) - which absorb carbon dioxide through photosynthesis as they grow. Periodically, these crops are harvested, processed for worldwide travel and shipped around the globe before finally being combusted in thermal power stations. The $\mathrm{CO}_{2}$ is then stripped from the waste gases, compressed (almost to a liquid), pumped through large pipes over potentially very long distances and finally stored deep underground in various geological formations (from exhausted oil and gas reservoirs through to saline aquifers) for a millennium or so.

The unquestioned reliance on negative-emission technologies to deliver on the Paris goals is the greatest threat to the new agreement. Yet BECCS, or even negative-emission technologies, received no direct reference throughout the 32-page package. Despite this, the framing of the $2^{\circ} \mathrm{C}$ goal and, even more, the $1.5^{\circ} \mathrm{C}$ one, is premised on the massive uptake of BECCS some time in the latter half of the century. Disturbingly, this is also the case for most of the temperature estimates ascribed to the outcome of the voluntary emissions cuts made by nations before the Paris meeting.

The scale of the assumption is breathtaking. It would be the equivalent of decades of planting and harvesting of energy crops over an area of

\section{$\rightarrow$ NATURE.COM}

Discuss this article online at:

go.nature.com/pmyruf one to three times that of India. At the same time, the aviation industry envisages powering its planes with biofuel, the shipping industry is seriously considering biomass to propel its ships and the chemical sector sees biomass as a potential feedstock - and by then there will be 9 billion or so human mouths to feed. This crucial assumption deserves wider scrutiny.

Relying on the promise of industrial-scale negative-emissions technologies to balance the carbon budget was not the only option available in Paris - at least in relation to $2^{\circ} \mathrm{C}$.

Reducing emissions in line with $2^{\circ} \mathrm{C}$ remains a viable goal - just. But rather than rely on post-2050 BECCS, deciding to pursue this alternative approach would have begged profound political, economic and social questions. Questions that undermine a decade of mathematically nebulous green-growth and win-win rhetoric, and questions that the politicians have decided cannot be asked.

Move away from the cosy tenets of contemporary economics and a suite of alternative measures comes into focus. Technologies, behaviours and habits that feed energy demand are all amenable to significant and rapid change. Combine this with an understanding that just $10 \%$ of the population is responsible for $50 \%$ of emissions, and the rate and scope of what is possible becomes evident.

The allying of deep and early reductions in energy demand with rapid substitution of fossil fuels by zero-carbon alternatives frames a $2^{\circ} \mathrm{C}$ agenda that does not rely on negative emissions. So why was this real opportunity muscled out by the economic bouncers in Paris? No doubt there are many elaborate and nuanced explanations but the headline reason is simple. In true Orwellian style, the political and economic dogma that has come to pervade all facets of society must not be questioned. For many years, green-growth oratory has quashed any voice with the audacity to suggest that the carbon budgets associated with $2{ }^{\circ} \mathrm{C}$ cannot be reconciled with the mantra of economic growth.

I was in Paris, and there was a real sense of unease among many scientists present. The almost euphoric atmosphere that accompanied the circulation of the various drafts could not be squared with their content. Desperate to maintain order, a club of senior figures and influential handlers briefed against those who dared to say so - just look at some of the Twitter discussions!

It is pantomime season and the world has just gambled its future on the appearance in a puff of smoke of a carbon-sucking fairy godmother. The Paris agreement is a road map to a better future? Oh no it's not.

Kevin Anderson is deputy director of the Tyndall Centre for Climate Change Research, UK. e-mail:kevin.anderson@manchester.ac.uk.Twitter@KevinClimate 